\section{Ganzheitliche Palliativmedizin am Lebensende}

\author{
In einem systematischen Review wurden empirisch validierte Modelle für \\ ganzheitliche Palliativtherapiestrategien bei Krebs und chronischen Erkran- \\ kungen in Europa untersucht.
}

Eations ne gut durchgeführte Palliativpflege am Ende des Lebens kann Schmerzen und andere Beschwerden lindern, seelischen Beistand leisten und eine gute Lebensqualität erhalten. Die Weltgesundheitsorganisation (WHO) empfiehlt daher, die Palliativmedizin zu einem festen Bestandteil der Gesundheitsversorgung zu machen und allen Patienten, die an einer zum Tode führenden Erkrankung leiden, anzubieten.

In dem aktuellen Review wurden insgesamt 14 Studien berücksichtigt. In sieben Studien wurden Modelle zu chronischen Erkrankungen (Demenz, Multiple Sklerose, chronische Herzinsuffizienz, HIV/AIDS und fortgeschrittene chronische Erkrankungen) evaluiert, in vier Studien ging es um die integrative Pflege in der Onkologie, drei Studien beschäftigten sich mit malignen und chronischen Erkrankungen, die zum Tode führten. Die meisten Studien kamen aus Großbritannien (sechs), zwei aus Spanien, zwei aus den Niederlanden und jeweils eine aus Italien, Deutschland, Norwegen und Frankreich.

Die Ergebnisse von 13 der 14 Studien erbrachten eindeutige Vorteile für die Anwendung ganzheitlicher Palliativmedizin: bessere Symptomkontrolle, eine geringere Belastung für die Pflegenden, Verbesserungen hinsichtlich Kontinuität und Koordination der Pflege, seltenere Klinikeinweisungen und Kostenersparnis. Auch verstarben die Patienten häufiger dort, wo sie es wünschten. Keine einzige der untersuchten Studien konnte ein in allen Punkten optimal funktionierendes Modell präsentieren.

Nach Ansicht der Reviewautoren kristallisierten sich folgende Parameter als entscheidend für eine gut funktionierende Palliativmedizin heraus:

- Intervention, die auf die Behandlung von Symptomen wie zum Beispiel
Dyspnoe, Schmerzen, Verstopfung, Erbrechen, Übelkeit, Diarrhoe und den Umgang mit psychischen Beschwerden wie Angst, Konfusion, Delirium zielt und die Bedürfnisse der Patienten und Angehörigen berücksichtigt.

_-Der (individuell) richtige Zeitpunkt, an dem mit der Palliativpflege begonnen wird.

_Funktionierende Rahmenbedingungen für ein multidisziplinäres Team von Ärzten, Pflegenden, Psychologen, Sozialarbeitern und Verwaltungsmitarbeitern, die für die Betreuung Sterbender ausgebildet sind.

Fazit: Eine gut durchgeführte ganzheitliche Palliativmedizin mit gut ausgebildetem interdisziplinär arbeitendem Personal kann die Situation von Patienten mit einer chronischen Erkrankung an ihrem Lebensende erheblich verbessern.

Kathrin von Kieseritzky

Siouta N et al. Integrated palliative care in Europe: a qualitative systematic literature review of empirically-tested models in cancer and chronic disease. BMC Palliative Care 2016;15:56

\title{
Mit Pregabalin die Radiotherapie unterstützen?
}

\section{Kann mit einem Wirkstoff, der an einen Kalziumkanal bindet, die Schmerz- übertragung gehemmt und die Wirkung einer Radiotherapie bei metastasen- bedingten Knochenschmerzen verstärkt werden?}

G oldstandard in der Therapie von tumorbedingten Knochenschmerzen ist die Radiotherapie. Doch nur bei etwa jedem fünften Patienten lassen sich die Schmerzen damit vollständig beseitigen. Tierexperimentelle Befunde, wonach Substanzen wie Pregabalin und Gabapentin die Schmerzübertragung hemmen und so die Wirkung der Strahlentherapie unterstützen könnten, wurden nun in einer randomisierten Studie klinisch getestet. 233 Patienten mit radiologisch nachweisbaren Knochenmetastasen erhielten randomisiert eine Radiotherapie (8 Gy in 1 oder 20 Gy in 5 Fraktionen) plus Pregabalin $(\mathrm{n}=116)$ oder
Placebo $(\mathrm{n}=117) .35$ Tage lang nahmen die Patienten entweder zweimal täglich $75 \mathrm{mg}$ Pregabalin oder Placebo ein. Am 1., 8., 15. und 22. Tag wurde die Medikation nach einer Einschätzung der Analgesie angepasst. Primärer Endpunkt war das Therapieansprechen, also die Reduktion der Schmerzen um mindestens 2 Punkte bis Woche 4 bei mindestens stabiler Opioiddosis.

Am häufigsten litten die Teilnehmer unter einem Prostata-, Mamma- oder Lungenkarzinom. Im Pregabalin-Arm erreichten 45 Patienten $(38,8 \%)$ den primären Endpunkt, im Placeboarm 47 (40,2\%; Odds Ratio 1,07; $\mathrm{p}=0,816)$. Es gab zwischen den Behandlungsarmen keine signifikanten Unterschiede hinsichtlich Schmerzintensität, Beeinträchtigung von Aktivitäten durch Schmerzen oder Lebensqualität, wohl aber bezüglich Stimmung $(\mathrm{p}=0,031)$ und Durchbruchschmerzen $(\mathrm{p}=0,037)$ jeweils zugunsten der Therapie mit Pregabalin.

Fazit: Die Ergebnisse stützen nicht die Zugabe von Pregabalin zur Radiotherapie bei krebsbedingten Knochenschmerzen. Künftig sollte die Wirkung von Pregabalin bei Knochenschmerzen mit neuropathischer Komponente untersucht werden.

Kathrin von Kieseritzky

Fallon $\mathrm{M}$ et al. Randomized Double-Blind Trial of Pregabalin Versus Placebo in Conjunction With Palliative Radiotherapy for Cancer-Induced Bone Pain. J Clin Oncol 2016;34(6):550-6 